\title{
Garten-Kleinode des Luis Morera auf der Kanareninsel La Palma
}

\author{
Hilke Steinecke \& Peter Schubert
}

\section{Abstract}

Luis Morera is a well known artist on La Palma (Canary Islands). He designed many places and gardens. Famous gardens are the Plaza de la Glorieta in Las Manchas de Abajo and the park Antonio Gómez Felipe in Los Llanos. Characteristic features are mosaics of broken tiles. Favorite motives are animals and plants of the Canary Islands. Artistic elements, lava and Canary plants are combined in perfect harmony.

\section{Zusammenfassung}

Luis Morera ist ein bekannter Künstler auf der Kanareninsel La Palma. Er gestaltete viele Plätze und Gärten. Besonders gelungen sind die Plaza de la Glorieta in Las Manchas de Abajo und der Park Antonio Gómez Felipe in Los Llanos. Beliebt in seinen Werken sind Mosaike aus gebrochen Kacheln, die bevorzugt Tiere und Pflanzen der Kanaren darstellen. Künstlerische Elemente, Lava und Kanarische Pflanzen harmonieren perfekt miteinander.

\section{Der palmerische Künstler LuIs Morera} An verschiedenen Orten auf der Kanareninsel La Palma, der Isla Bonita, kann der Urlauber kleine Gärten und Plätze, Skulpturen oder gestaltete Fassaden entdecken, die einen ganz besonderen Stil aufweisen: Meist gehen dabei künstlerische Elemente eine perfekte Verbindung mit Aspekten

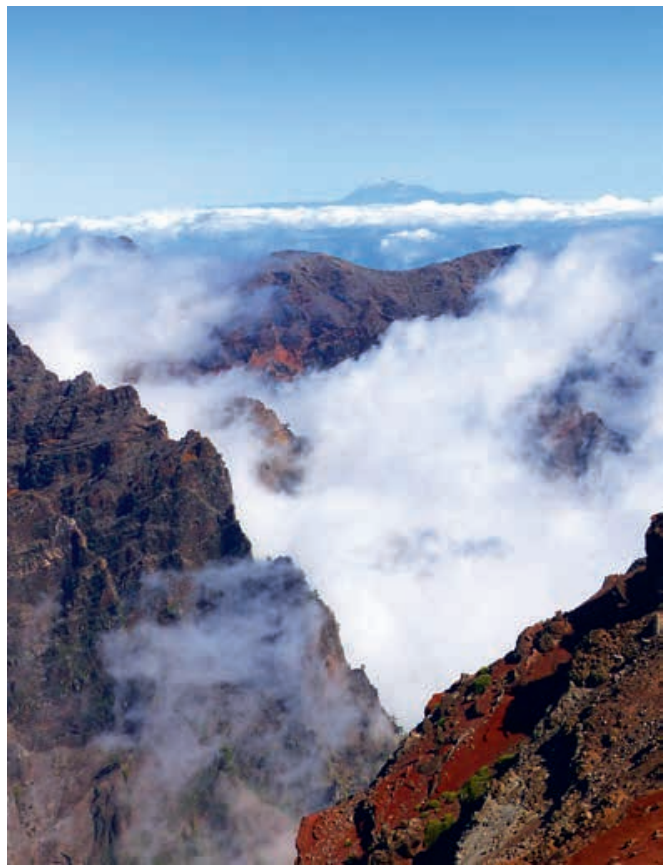

Abb. 1: Die wilde Landschaft von La Palma mit dem Einsturzkrater Caldera de Taburiente und dem Teide im Hintergrund hat den Künstler Morera inspiriert. (Foto: H. Steinecke) der Natur der Kanaren ein. Es werden natürliche Materialien verwendet. Bevorzugt werden dabei markante Lavasteine von La Palma für Mauern, Grotten oder Pergolen eingesetzt. Häufig spielen auch typische endemische Pflanzen und Tiere der Insel bei der Gestaltung eine wichtige Rolle. Auffällig sind die farbenfrohen Mosaike aus

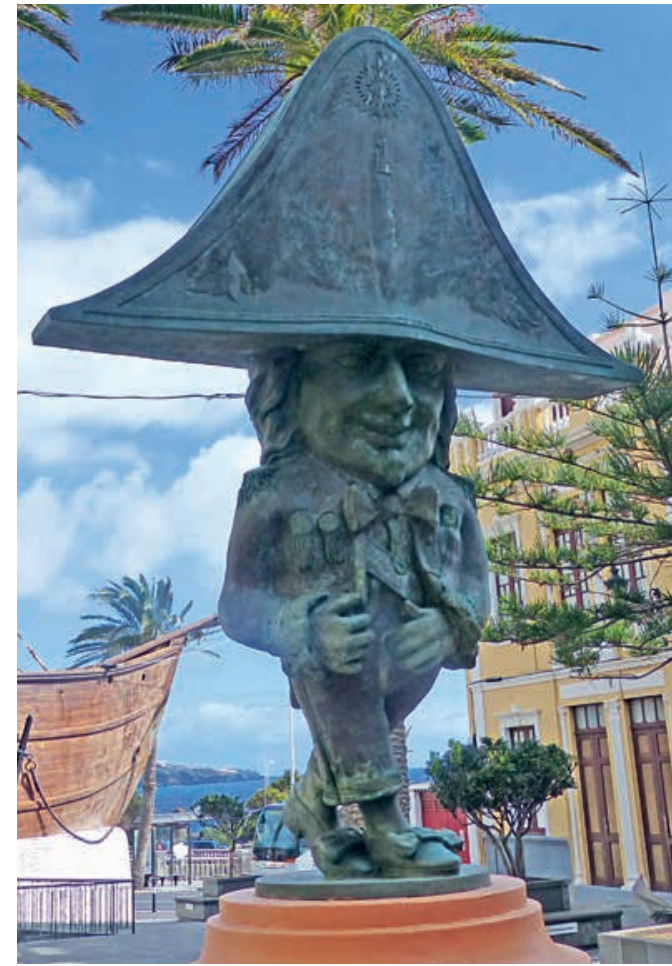

Abb. 2: Der Zwerg von Santa Cruz. (Foto: H. Steinecke) 
gebrochenen Kacheln, mit denen die Schönheit der Kanarischen Landschaft sowie der Flora und Fauna gewürdigt wird.

Geschaffen wurden diese Kunstwerke von Luis Morera, einem am 10. Oktober 1946 in der Inselhauptstadt Santa Cruz de La Palma geborenen Künstler, der heute als der Künstler Nummer 1 auf La Palma gilt. Schon als Jugendlicher stellte der vielseitig begabte Morera seine Bilder aus und gewann dafür Preise. Im Jahr 2001 wurde er von der Kanarischen Regierung für sein Gesamtkunstwerk mit der Goldmedaille ausgezeichnet. In den 1970er Jahren widmete sich der junge Morera intensiv der Musik: er engagierte sich als Sänger, Komponist sowie Texter und gründete die überregional bekannte Folkloregruppe Taburiente, benannt nach dem riesigen palmerischen Einsturzkrater Caldera de Taburiente. Bildende Kunst studierte Morera bei seinem späteren Freund CÉSAR MANRIQUe, einem weit bekannten Künstler von der Kanareninsel Lanzarote.

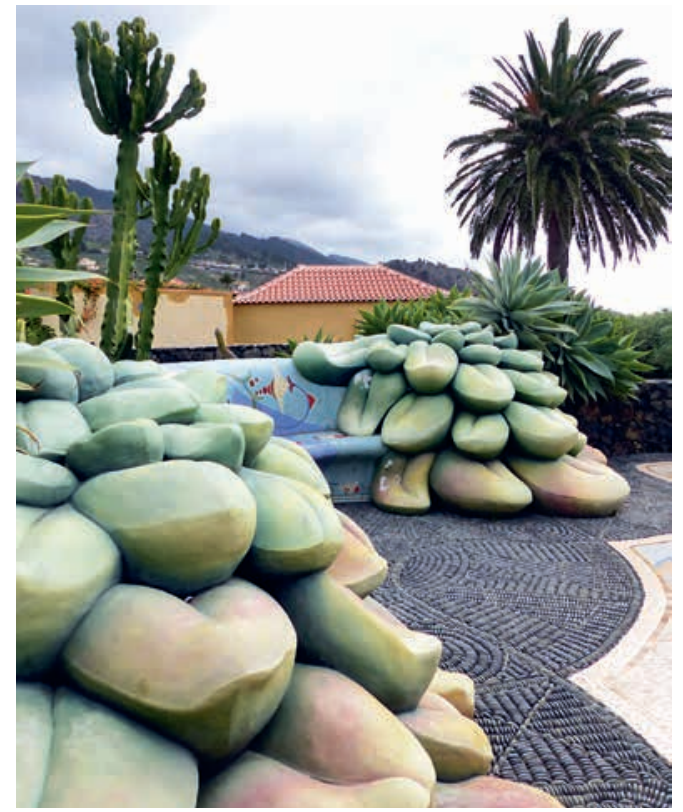

Abb. 3: Künstliche Rosetten von Aeonien aus Beton sowie echte Schwanenhalsagaven umgeben eine Bank an der Plaza de la Glorieta. (Foto: H. Steinecke)

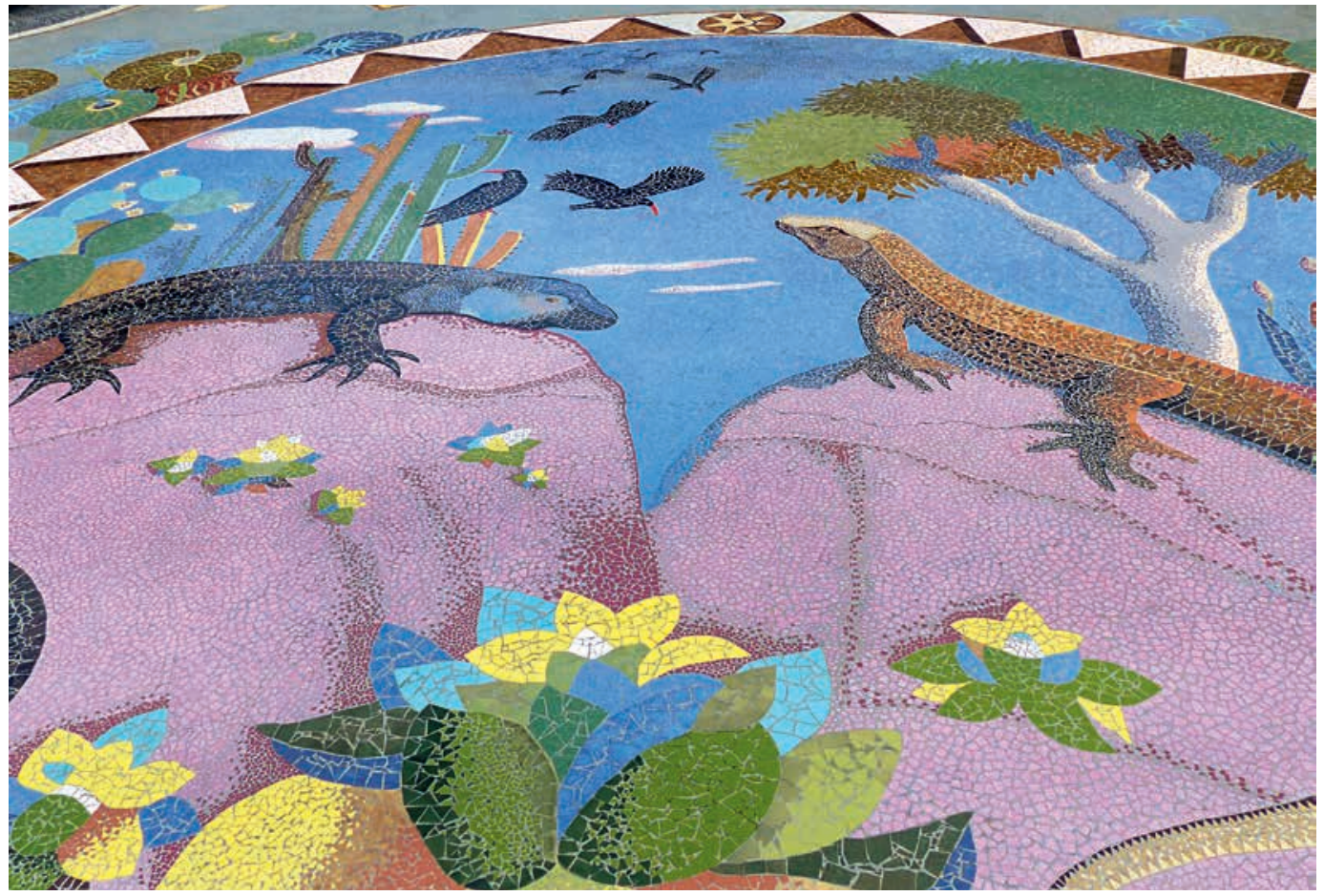

Abb. 4: Plaza de la Glorieta, Kachelmosaik mit Kanarischen Eidechsen, Alpen-Krähen, Kanarischer Wolfsmilch und Kanarischem Drachenbaum. (Foto: H. STEInecke) 
In Moreras Geburtsstadt Santa Cruz ist das Denkmal des Zwerges von Santa Cruz nicht zu übersehen. Mit dem Nachbau des Schiffes von Kolumbus (der Santa Maria) im Hintergrund ist der Zwerg mit großer Kopfbedeckung, die an den Hut von Napoleon erinnert, wohl eines der beliebtesten Fotomotive in der Stadt. Tatsächlich entstand die Zwergenfigur als Karikatur von NApoleon Bonaparte. Morera hat mit seiner Skulptur aus dem Jahre 2000 eine alte Tradition der Insulaner aufgegriffen, denn die ersten tanzenden Zwerge in spanischen Kleidern sind aus dem Jahr 1833 dokumentiert, als sie bei Feiern anlässlich der Krönung von Isabell II. erschienen.

\section{Plaza de la Glorieta}

In Las Manchas de Abajo, einem kleinen Ort der Gemeinde Los Llanos im Westen von La Palma, in der Nähe von Tazacorte, ist ein kleines, aber feines Werk des Künstlers zu bewundern. Eine Fläche von nur 300 Quadratmetern (das ist etwa



Abb. 5: Plaza de la Glorieta, eine Bank unter der Pergola wird von echten Blättern sowie Betonblättern einer Monstera umgeben. (Foto: H. Steinecke) die Hälfte der Fläche einer Ausstellungsgalerie im Palmengarten) nimmt der mit Kachelmosaiken verzierte runde Dorfplatz Plaza de la Glorieta ein. Ursprünglich sollte dieser Platz bzw. kleine Garten in der einwohnerstärksten Stadt von La Palma, in Los Llanos, angelegt werden, was aber auf keinen besonderen Zuspruch stieß. Als Ausweichort wurde der kleine, ländlich geprägte Ort Las Manchas de Abajo angeboten. Die Anlage der Plaza erfolgte in den Jahren 1993-1996.

Ein Teil der Fläche des Platzes ist mit einem aufwändigen Muster aus schwarzen, vom Meer abgerundeten Basaltsteinen gepflastert. Die Kachelmosaike auf dem Boden und den Sitzbänken zeigen typische Zier-, Wild- und Nutzpflanzen der Kanaren. Auch wenn sie stilisiert dargestellt sind, kann man die entsprechenden Arten eindeutig erkennen. Besonders prominent wirken dabei die Kanaren-Endemiten Drachenbaum (Dracaean drago) und Kanaren-Wolfsmilch (Euphorbia canariensis) sowie Bananenstauden, Feigenkakteen und

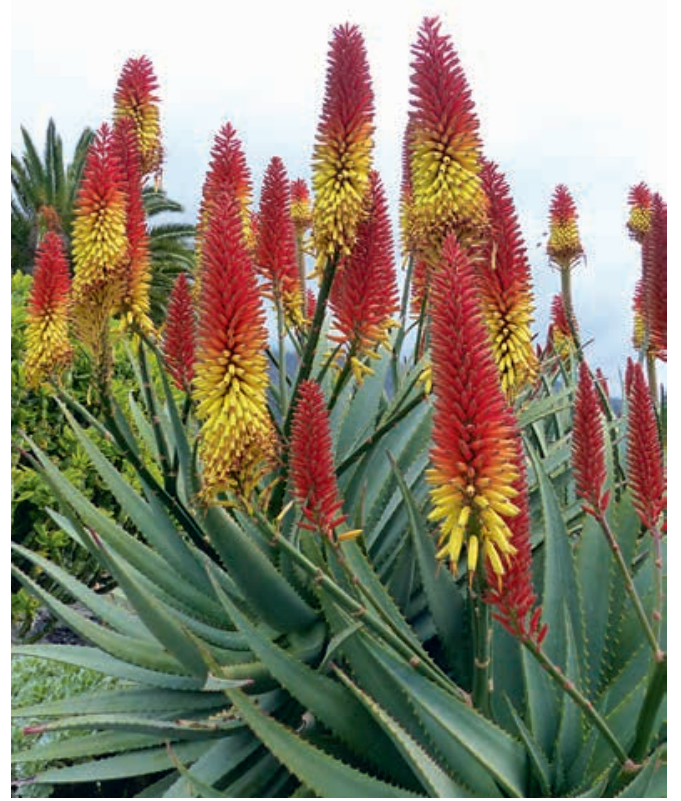

Abb. 6: Wie Sylvesterraketen wirken diese Aloe-Blütenstände, die sich hier um den Jahreswechsel besonders üppig zeigen. (Foto: H. Steinecke) 


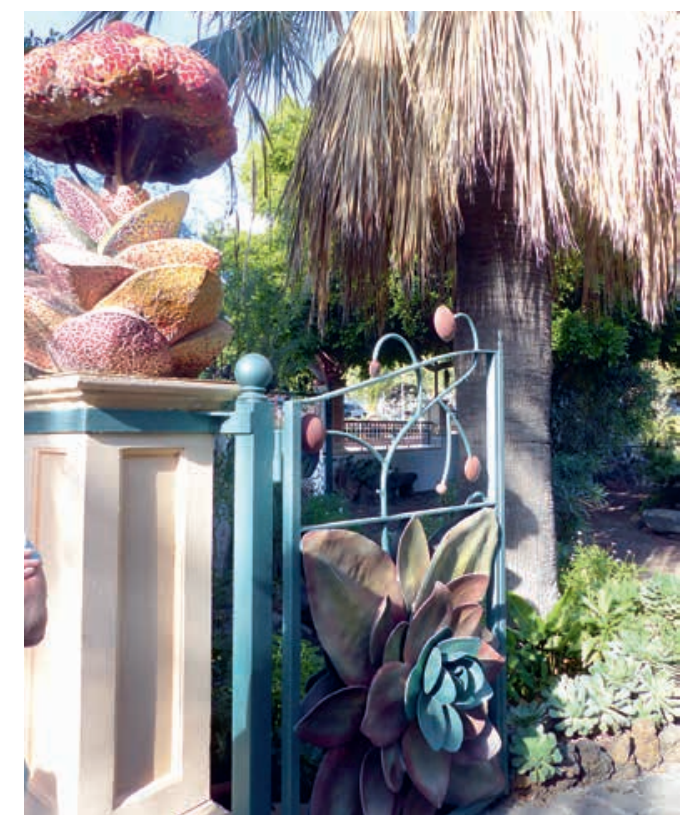

Abb. 7: Eingangstor zum Park Antonio Gómez Felipe, die floralen Verzierungen erinnern an Motive des Jugendstils. (Foto: H. STEINECKe)

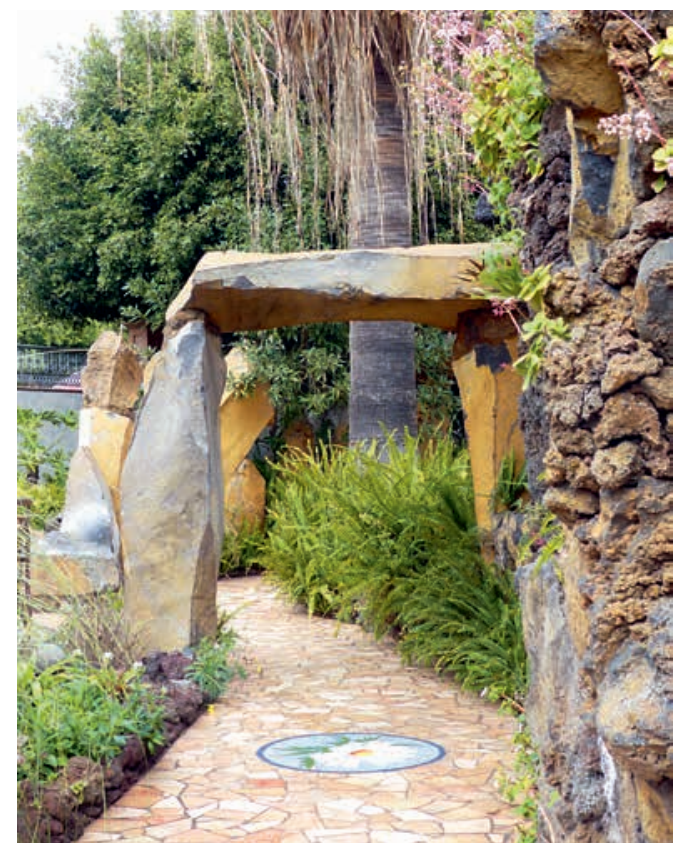

Abb. 8: Ein schmaler gewundener Pfad zieht sich an der Grotte vorbei durch den Garten. (Foto: H. STEinecke)

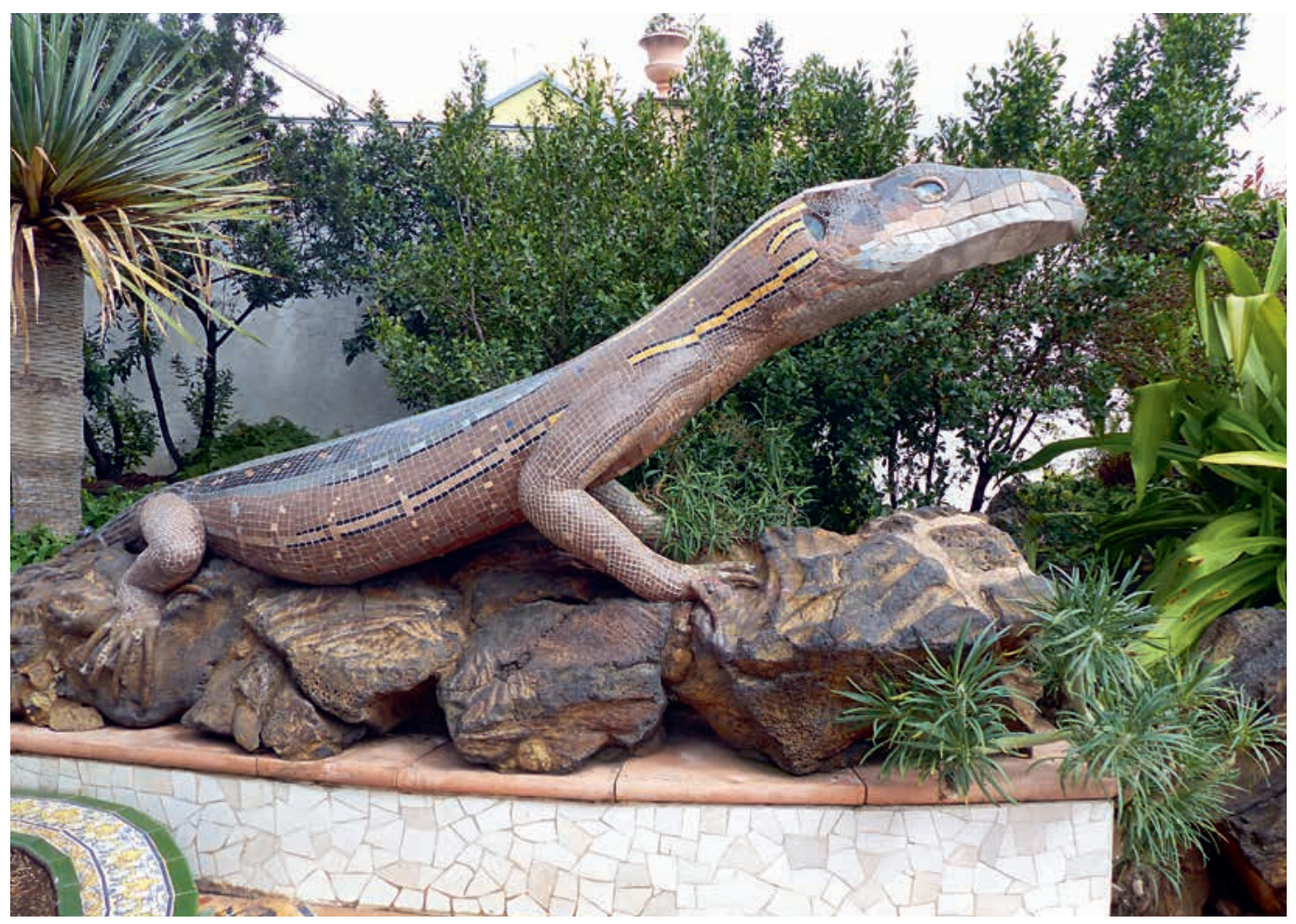

Abb. 9: Fast schon wie ein Saurier wirkt diese überlebensgroße Kanarische Eidechse mit Mosaik-Oberfläche.

(Foto: H. Steinecke) 


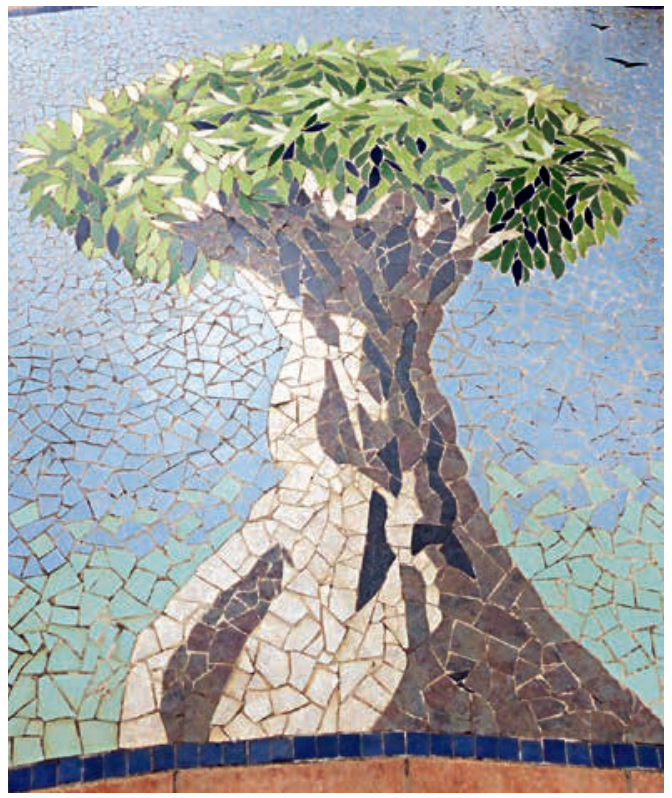

Abb. 10: Kanarischer Drachenbaum in Mosaiktechnik im Park Antonio Gómez Felipe. (Foto: H. Steinecke)

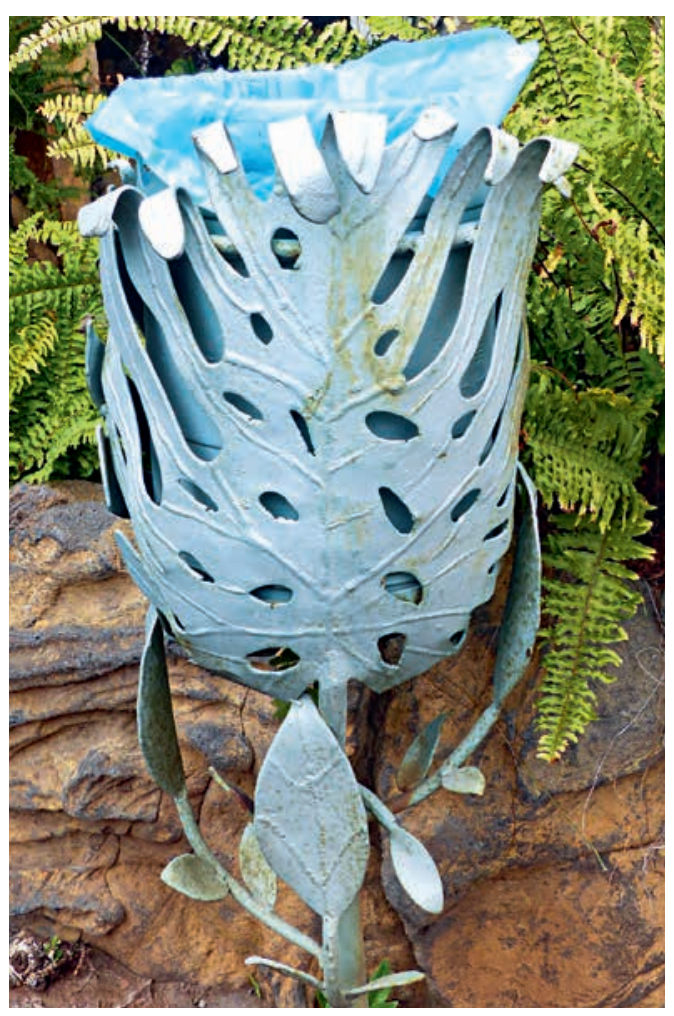

Abb. 11: Abfalleimer in der Form eines Monstera-Blattes. (Foto: H. STEINECKe)
Strelitzien. Nicht zu übersehen sind im Mosaikboden zwei Kanaren-Eidechsen in der für La Palma endemischen Unterart (Gallotia galloti palmae). Diese Eidechsen sind auf La Palma überall häufig anzutreffen. Die Tiere wärmen sich oft auf steinigen Mauern bzw. Dächern auf oder warten an touristischen Punkten darauf, dass für sie ein paar Leckereien abfallen. Über den Mosaik-Eidechsen sind Alpen-Krähen (Pyrrhocorax pyrrhocorax) dargestellt. Die Alpenkrähe ist ein Rabenvogel mit einem charakteristischen sichelförmig gebogenen roten Schnabel. Sie gilt als „Wappenvogel“ von La Palma. Nicht zu verwechseln ist sie mit dem viel größeren Kolkraben (Corvus corax), den man häufig in der Caldera de Taburiente antreffen kann. Auch ein überlebensgroßes Chamäleon in Mosaik-Technik lädt zum genaueren Betrachten ein. Das Tier aus Stein sitzt in einem Wasserbecken, das von Stricklava-Brocken eingefasst wird.

Die Geschwungenen Formen des Gartens, Farbgebung und Verwendung von Mosaiken erinnern an die Arbeiten des spanischen Architekten AnToNi Gaudí (1852-1926), von dem sich Morera vermutlich hat inspirieren lassen. Moreras Motto ist es, die Identität und Ursprünglichkeit von La Palma zu verteidigen, die Natur zu achten und zu bewahren. Bewusst verwendet er deshalb Naturmaterialien (z. B. Lavasteine) von der Insel (s. o.). Seine Kunstformen lässt er mit Formen der Natur harmonisch verschmelzen. Besonders gelungen ist dies an einer Sitzbank unter einer Pergola. Sie wird von echten Monstera-Blättern umwachsen. Diese gehen dann aber kontinuierlich in künstliche, aus Beton gegossene und grün angemalte Monste$r a$-Blätter über. Beeindruckend sind auch zwei riesige Beton-Aeonien, die sich links und rechts einer anderen mit Mosaiken verzierten Bank befinden. Die in der Nähe wachsenden großen Blattrosetten von Schwanenhals-Agaven (Agave attenuata) greifen die Blattrosettenform ,in echt“ auf. Pflanzen und Kunst gehen in diesem kleinen Garten eine perfekte Verbindung miteinander ein.

Auf einem mittleren, von Lavasteinen begrenzten Beet sind neben diversen Sukkulenten auch Endemiten von La Palma gepflanzt, so auch die beiden strauchigen Natternkopf-Arten (Echi- 




Abb. 12: Strauchiger Natternkopf (Echium) mit ungewöhnlicher Verbänderung. (Foto: H. STEINEcke)

um brevirame und $E$. webbii) sowie verschiedene Arten an Strauch-Margeriten (Argyranthemum). Auf dem Beet an der Außenbegrenzung des Gartens fallen ein großer Feigenkaktus sowie stattliche Aloen auf. Gerade um Weihnachten öffnen viele tropische und subtropische Pflanzen ihre mitunter auffälligen großen Blüten. Die rot-gelben Blütenstände von Aloe-Hybriden wirken wie riesige Fackeln oder Feuerwerksraketen.

\section{Der Park Antonio Gómez Felipe}

Ein weiterer kleiner, etwa 6000 Quadratmeter umfassender Morera-Garten befindet sich südlich der Innenstadt von Los Llanos. Ursprünge dieses öffentlichen Parks stammen aus dem Jahr 1958. Er wurde damals vom Zahnarzt und Kunstsammler Antonio Gómez Felipe aus Los Llanos den Bürgern seiner Stadt gestiftet, weshalb der Park seinen Namen trägt. Da hier einige botanische Besonderheiten gepflanzt wurden, lautet der ausführliche Name des Parks El pequeño jardín botánico del parque Antonio Gómez Felipe. In 1990 begannen Umbau- und Sanierungsarbeiten, die

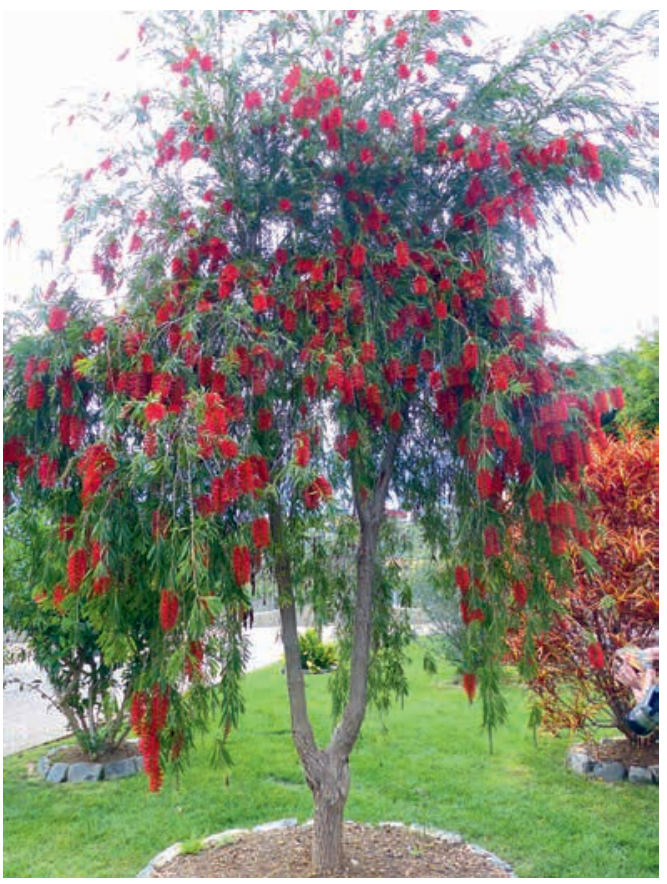

Abb. 13: Üppig blühender Zylinderputzerstrauch (Callistemon) im formalen Bereich des Parks. (Foto: H. Steinecke)

sich über 20 Jahre hinzogen, bis der neue Park schließlich im Jahr 2010 wiedereröffnet wurde. Vielleicht dauerten die Arbeiten so lange, weil sie im Rahmen von sozialen Projekten von arbeitslosen Jugendlichen durchgeführt wurden. Der Park besteht nun aus zwei Teilen.

Der obere, neue Teil wurde von Luis MoreRa und Natan Teutsch entworfen. Er zeigt ein wunderbares Zusammenspiel zwischen Kunst, palmerischer Natur und der Pflanzenwelt der Kanaren. Von der Straße kommend führt eine Treppe direkt in den Garten hinab, wo zwei mittlerweile recht große Kanarische Dattelpalmen (Phoenix canariensis) auffallen. Seitlich sitzen zwei überdimensional große steinerne Kanaren-Eidechsen, deren Oberfläche mit unzähligen kleinen Mosaiksteinchen überzogen ist. Durch die unterschiedlichen Höhenniveaus gibt es von verschiedenen Blickwinkeln immer wieder neue Perspektiven und dementsprechend Neues zu entdecken. Auf einem gebogenen Weg schlängelt man sich an einer Natursteinbrücke, einem kleinen Wasserfall oder einer farnbewachsenen Lavagrotte vorbei. 


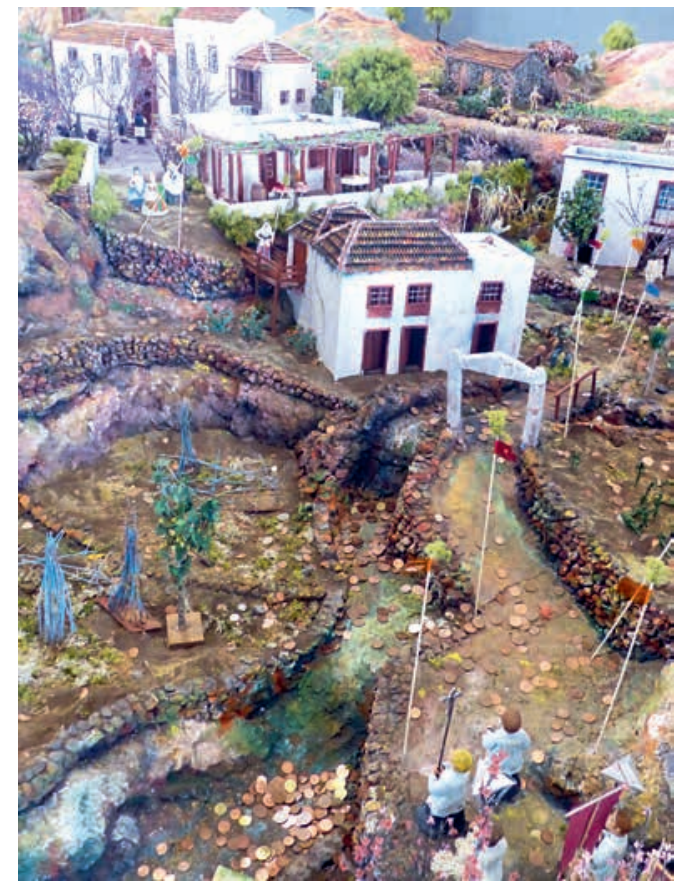

Abb. 14: Krippenlandschaft Los Llanos zu Weihnachten 2018. Jedes Jahr wird sie neu gestaltet, für die ersten war Morera verantwortlich. (Foto: H. STeinecke)

Dazwischen wachsen verschiedenste Endemiten der Kanaren, so auch die auffälligen strauchigen Natternköpfe (Echium) und Aeonien. Und fast an jeder Ecke stößt man auf florale Formen aus Künstlerhand. Nicht zu übersehen ist die Ähnlichkeit mit Motiven des Jugendstils. So zeigt ein Gittertor große stilisierte Aeonien. Direkt daneben gedeihen die lebenden Pflanzenvorbilder. Liebevoll sind auch die Abfalleimer in Form von Monstera-Blättern gestaltet. Als Inspiration für diesen Garten diente vor allem das bekannte Tryptichon „Der Garten der Lüste“ des niederländischen Malers Hieronymus Bosch (etwa 1450-1516), das im Madrider Museo del Prado zu bewundern ist. Auf der linken Tafel des Tryptichons ist der Garten Eden dargestellt. Hier ist hinter Adam ein Drachenbaum zu erkennen. Durch dieses Gemälde wurde der Kanarische Drachenbaum überhaupt erst fernab der Kanaren bekannt.

Der untere Teil des Parks zeigt rechteckige Rasenflächen und gerade Wege. Hier gedeihen einige schöne Gehölze wie Roter Pfefferbaum
(Schinus molle), Silbereiche (Grevillea argentea), Pinselstrauch (Callistemon) oder Araukarien (Araucaria heterophylla). Dazwischen sind Spielgeräte für Kinder aufgestellt.

Wer in der Weihnachtszeit durch die Altstadt von Los Llanos bummelt, kann noch ein weiteres Kleinod entdecken, das im Zusammenhang mit Luis Morera steht. Denn seit 1991 wird jedes Jahr im Untergeschoss der Casa de la Cultura eine Krippe ausgestellt. Es ist keine einfache Krippe, sondern eine Modelllandschaft, in der, jedes Jahr anders, eine kanarische Landschaft und das dortige ländliche Leben mit wiedererkennbaren Gebäuden thematisiert wird. Diese speziellen Krippen werden Belén oder Nacimientos genannt. Im Mittelpunkt stehen die Heiligen Drei Könige auf dem Wege zum Jesuskind. Niemals fehlen darf aber auch eine Figur, die versteckt in einer Ecke ihre Notdurft verrichtet, quasi als Gag und Suchspiel für die Kinder. In den ersten 15 Jahren war Luis Morera für diese Krippenlandschaft verantwortlich. Jetzt sind es vor allem zwei Stadtgärtner von Los Llanos, die diese Tradition weiterführen.

\section{Internetseiten}

https://de.wikipedia.org/wiki/Luis_Morera https://de.wikipedia.org/wiki/Plaza_de_La_Glorieta http://mehr-als-eine-insel.blogspot.com/2013/08/park-antonio-gomez-felipe-in-los-llanos.html

https://www.la-palma24.info/weihnachtskrippe-in-los-llanos/

\section{Anschriften der Autorin und des Autors}

Dr. Hilke Steinecke, Palmengarten Frankfurt, Siesmayerstraße 61, 60323 Frankfurt, E-Mail: hilke.steinecke@stadt-frankfurt.de

Dr. Peter Schubert, Universität Mainz, FB Biologie, Gresemundweg 2, 55128 Mainz,

E-Mail: schubepe@uni-mainz.de 